\title{
Topological relations between three-periodic nets. II. Binodal nets
}

\author{
Vladislav A. Blatov and Davide M. Proserpio
}

Acta Cryst. (2009). A65, 202-212

Copyright (C) International Union of Crystallography

Author(s) of this paper may load this reprint on their own web site or institutional repository provided that this cover page is retained. Republication of this article or its storage in electronic databases other than as specified above is not permitted without prior permission in writing from the IUCr.

For further information see http://journals.iucr.org/services/authorrights.html

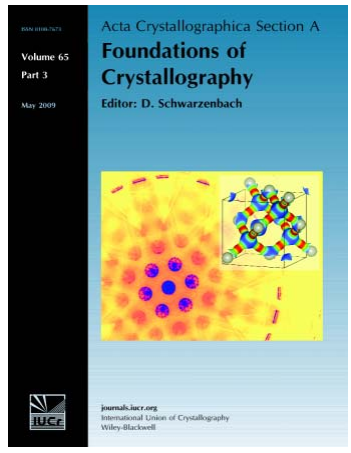

Acta Crystallographica Section A: Foundations of Crystallography covers theoretical and fundamental aspects of the structure of matter. The journal is the prime forum for research in diffraction physics and the theory of crystallographic structure determination by diffraction methods using X-rays, neutrons and electrons. The structures include periodic and aperiodic crystals, and non-periodic disordered materials, and the corresponding Bragg, satellite and diffuse scattering, thermal motion and symmetry aspects. Spatial resolutions range from the subatomic domain in charge-density studies to nanodimensional imperfections such as dislocations and twin walls. The chemistry encompasses metals, alloys, and inorganic, organic and biological materials. Structure prediction and properties such as the theory of phase transformations are also covered.

\section{Crystallography Journals Online is available from journals.iucr.org}


Acta Crystallographica Section A

Foundations of

Crystallography

ISSN 0108-7673

Received 13 January 2009

Accepted 19 February 2009

\section{Topological relations between three-periodic nets. II. Binodal nets}

\author{
Vladislav A. Blatov ${ }^{a}$ and Davide M. Proserpio \\ ${ }^{\mathbf{a}}$ Samara State University, Ac. Pavlov St. 1, Samara 443011, Russia, and ${ }^{\mathbf{b}}$ Dipartimento di Chimica \\ Strutturale e Stereochimica Inorganica (DCSSI), Università di Milano, Via G. Venezian 21, 20133 \\ Milano, Italy. Correspondence e-mail: blatov@ssu.samara.ru
}

\begin{abstract}
The TOPOS program package was used to generate all subnets of 3- to 12coordinated binodal nets taken from the Reticular Chemistry Structure Resource database. 38304 binodal nets with novel topologies were revealed and stored in the TTD collection. A new invariant, the adjacency matrix of the shell graph of a node, is proposed to distinguish the node local topology. With this invariant, the first six examples of binodal-quasi-uninodal nets were discovered. 4604 organic and metal-organic frameworks were analyzed to find examples of the topologies generated. It was shown that many edge-transitive nets as well as unknown topologies occur in crystal structures.
\end{abstract}

(C) 2009 International Union of Crystallography Printed in Singapore - all rights reserved connected graph] (Delgado-Friedrichs et al., 2005). Incidentally, nets with a lower periodicity, well known to mathematicians (Grünbaum \& Shephard, 1987), have been studied much less in crystal chemistry (Koch \& Fischer, 1978; O'Keeffe \& Hyde, 1980; O'Keeffe, 1992).

O'Keeffe has gathered many nets crucial for crystal chemistry since the beginning of the century in the RCSR database (Ockwig et al., 2005; O'Keeffe et al., 2008). This work has encouraged systematic investigations of crystal-structure topologies in organic, inorganic and metal-organic compounds (Blatov et al., 2004; Baburin et al., 2005, 2008a,b; Blatov \& Peskov, 2006; Baburin \& Blatov, 2007; Baburin, 2008). Most of the results of these studies are collected in the TTO database, although the number of structures processed is less than 5000 . However, this now allows one to draw some conclusions about the occurrence of various topologies. Thus, in valence-bonded interpenetrated inorganic and metal-organic frameworks, as well as in single metal-organic frameworks, the three most preferred topological motifs ${ }^{1}$ are dia $(4 / 6 / c 1)$, pcu $(6 / 4 / c 1)$ and srs (3/10/c1) (Blatov et al., 2004; Ockwig et al., 2005; Baburin et $a l ., 2005)$. In organic molecular crystals with hydrogen-bonded single networks the results are similar: dia, pcu, sxd (6/3/o1) and hex (8/3/h4) (Baburin \& Blatov, 2007); for hydrogenbonded coordination compounds they are pcu, bcu $(8 / 4 / c 1)$, hex and dia (Baburin, 2008; Baburin et al., 2008a,b). Thus, nature chooses the same topological motifs; moreover, all of them are sphere packings [cf. Koch et al. (2006) and references therein].

The question as to why some nets occur more frequently in crystals has still not been answered. O'Keeffe and co-workers (Ockwig et al., 2005; Delgado-Friedrichs et al., 2006, 2007)

\footnotetext{
${ }^{\mathbf{1}}$ Hereafter, the RCSR three-letter symbols (if available) are used to designate net topologies. Fischer's symbols $k / m / f n$ (Koch et al., 2006) are given for sphere packings along with the RCSR symbols.
} 
assumed that such nets should be topologically the simplest; in particular, they should have a minimal number of inequivalent nodes and/or edges as well as the highest possible symmetry at the nodes (we prefer the term 'node' instead of 'vertex' for nets). Indeed, the most frequent nets observed for metalorganic frameworks and hydrogen-bonded supramolecular assemblies are uninodal (vertex-transitive), i.e. have one kind of node, and all but hex and sxd are edge-transitive (with one kind of edge). However, many other uninodal and/or edgetransitive nets are not so abundant, so a close inspection of node symmetries is required to ascertain whether this rule is sufficient. The opposite question is important as well: why do some nets never or rarely occur in nature? To answer this question, the list of natural nets should be extended with artificial nets generated by some tailored methods. In this case, the methods for producing the nets both $a b$ initio and in relation to the natural nets are important. The largest project for generating $a b$ initio nets is EPINET (Hyde et al., 2006; Ramsden et al., 2009) where three-periodic nets are derived from two-dimensional hyperbolic tilings irrelevant to real crystal structures. Studying the occurrence of EPINET nets shows how stochastic the realization of topological motifs in crystals is. However, nobody has yet performed such an analysis. The most exciting example of producing artificial nets related to real crystal structures is the Atlas of Hypothetical Zeolite Frameworks [http://www.hypotheticalzeolites.net (Treacy et al., 2004; Delgado-Friedrichs \& O'Keeffe, 2005)]. In this case, the generated nets are to obey some geometrical criteria relevant to zeolite structures.

In the first paper of this series, Blatov (2007) proposed one more method for obtaining new nets that are possibly relevant to crystal structures. Starting from a frequently occurring net in a maximum-symmetry Euclidean embedding and considering all possible ways of decreasing the symmetry and coordination of the net, one can obtain all of its subnets with a given number of inequivalent nodes. With a tailored procedure of the TOPOS program package, Blatov (2007) collected all 5278 uninodal subnets of the RCSR uninodal nets. Some of

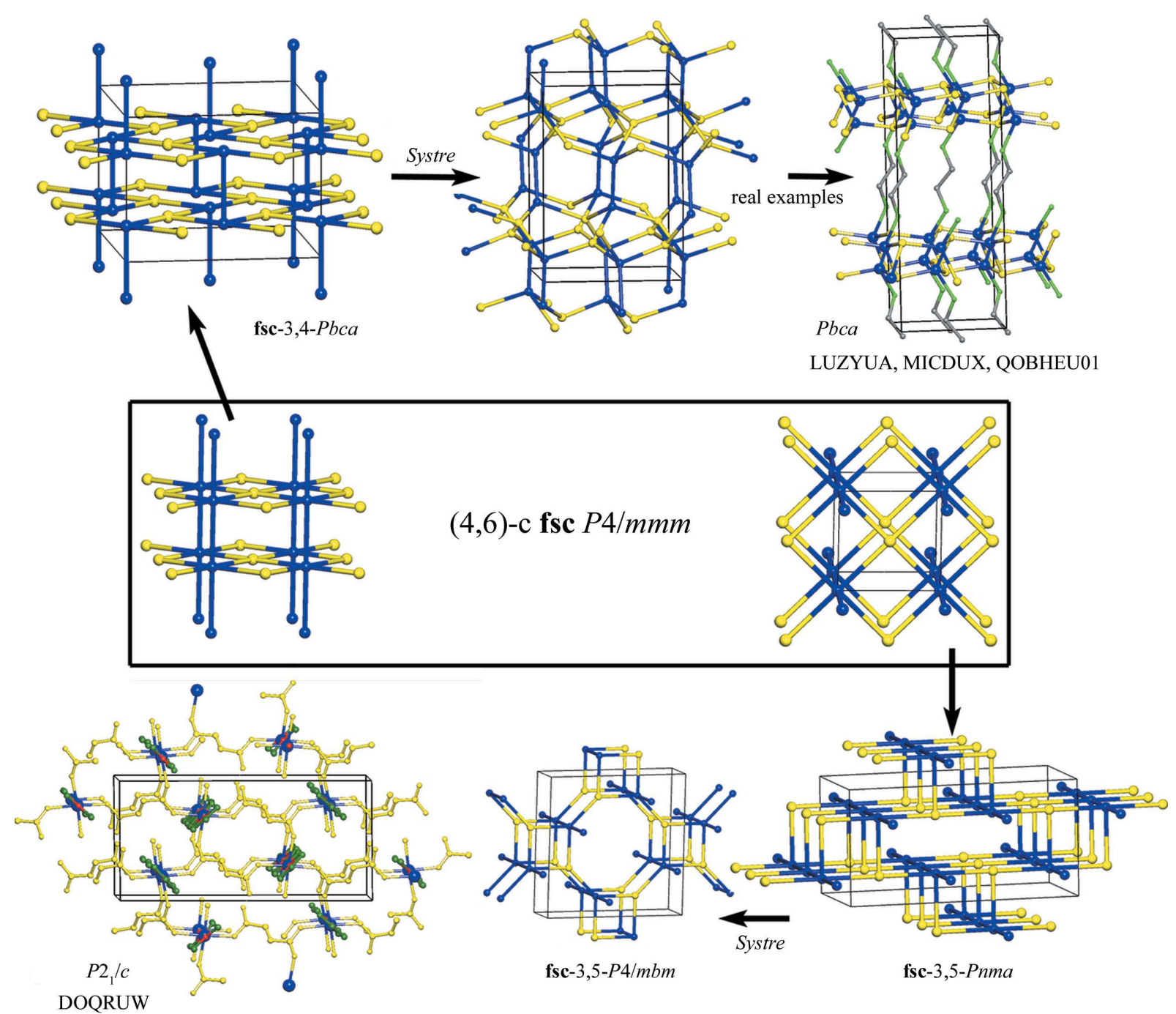

Figure 1

The procedure for deriving two subnets $(3,4)$ and $(3,5)$-coordinated from $(4,6)$-fsc. For each subnet (top left and bottom right) we also show the Systreoptimized embedding (center top and bottom) and one real example [LUZYUA, MICDUX and QOBHEU10 are $\mathrm{Zn}_{2} X_{2}($ ethylenediamine), $X=\mathrm{S}$, Se, Te; DOQRUW is $\mathrm{Mn}\left(\mathrm{H}_{2} \mathrm{O}\right)$ (fumarato)(4,4'-bipy)]. See text for further details. 
Table 1

The 225 EPINET nets inequivalent to RCSR nets found among the subnets generated.

\begin{tabular}{|c|c|c|}
\hline $\begin{array}{l}\text { Node } \\
\text { degrees }\end{array}$ & No. & Net name \\
\hline 3,3 & 35 & $\begin{array}{l}\text { sqc326, sqc946, sqc3051, sqc3053, sqc3058, sqc5599, sqc5600, sqc5601, sqc5602, sqc9243, sqc9247, sqc9248, sqc9250, sqc9252, sqc9264, } \\
\text { sqc9267, sqc11250, sqc11253, sqc11254, sqc11255, sqc11257, sqc11261, sqc12871, sqc12874, sqc12881, sqc13759, sqc13771, sqc13774, } \\
\text { sqc13776, sqc14290, sqc14292, sqc14293, sqc14294, sqc14297, sqc14298 }\end{array}$ \\
\hline 3,4 & 90 & $\begin{array}{l}\text { sqc74, sqc185, sqc188, sqc476, sqc515, sqc519, sqc522, sqc1255, sqc1374, sqc1383, sqc1388, sqc1425, sqc1426, sqc1427, sqc1429, sqc1431, } \\
\text { sqc1436, sqc1441, sqc2196, sqc2205, sqc2209, sqc3681, sqc3705, sqc3852, sqc3853, sqc3880, sqc3881, sqc3890, sqc3895, sqc3896, } \\
\text { sqc4674, sqc4709, sqc5594, sqc6667, sqc6855, sqc6856, sqc6930, sqc6934, sqc7386, sqc8168, sqc8169, sqc8170, sqc8171, sqc } 9849, \\
\text { sqc } 9903, s q c 9904, s q c 9912, s q c 9916, s q c 10089, s q c 10093, s q c 10103, s q c 10603, s q c 10611, s q c 10615, s q c 10660, s q c 10667, s q 1155, \\
\text { sqc11240, sqc11241, sqc11249, sqc11984, sqc12015, sqc12018, sqc12019, sqc12020, sqc12028, sqc12029, sqc12251, sqc12269, sqc12277, } \\
\text { sqc13150, sqc13541, sqc13993, sqc13996, sqc13997, sqc14002, sqc14004, sqc14015, sqc14016, sqc14018, sqc14019, sqc14134, sqc14135, } \\
\text { sqc14139, sqc14149, sqc14150, sqc14389, sqc14390, sqc14396, sqc14397 }\end{array}$ \\
\hline 3,5 & 19 & $\begin{array}{l}\text { sqc138, sqc689, sqc707, sqc1743, sqc1744, sqc2115, sqc2154, sqc2281, sqc2155, sqc5478, sqc5941, sqc7845, sqc7846, sqc8008, sqc8009, } \\
\quad s q c 8010, s q c 8011, s q c 8013, s q c 12425\end{array}$ \\
\hline 3,6 & 14 & $s q c 27, s q c 251, s q c 293, s q c 294, s q c 892, s q c 893, s q c 962, s q c 2403, s q c 2983, s q c 2984, s q c 2985, s q c 5381, s q c 8925, s q c 11083$ \\
\hline 3,8 & 2 & sqc494, sqc7928 \\
\hline 4,4 & 19 & $\begin{array}{l}\text { sqc466, sqc } 967, \text { sqc2186, sqc2188, sqc2189, sqc3818, sqc3848, sqc3868, sqc5553, sqc5567, sqc8092, sqc8105, sqc8112, sqc13484, sqc13489, } \\
\quad \text { sqc13496, sqc13497, sqc13505, sqc13511 }\end{array}$ \\
\hline 4,5 & 19 & $\begin{array}{l}\text { sqc278, sqc280, sqc847, sqc932, sqc955, sqc1101, sqc1253, sqc2481, sqc2482, sqc2484, sqc2644, sqc2996, sqc3986, sqc3989, sqc5464, } \\
\quad s q c 6809, s q c 6814, s q c 6815, s q c 9092\end{array}$ \\
\hline 4,6 & 12 & $s q c 374, s q c 502, s q c 645, s q c 1245, s q c 1730, s q c 3754, s q c 3793, s q c 4582, s q c 4588, s q c 4595, s q c 4596, s q c 6738$ \\
\hline 4,7 & 1 & sqc1858 \\
\hline 4,8 & 3 & sqc934, sqc1964, sqc1990 \\
\hline 5,5 & 3 & sqc3254, sqc5617, sqc7317 \\
\hline 5,6 & 3 & sqc1067, sqc7877, sqc8329 \\
\hline 5,8 & 3 & sqc1330, sqc6767, sqc6771 \\
\hline 6,8 & 2 & sqc1854, sqc1954 \\
\hline
\end{tabular}

the novel nets were shown to be of potential interest for crystal chemistry. At that time, there were no data for their occurrence and other important RCSR nets, binodal and edgetransitive nets, were not treated. In this paper we will consider these two classes of nets with particular interest in their realization in nature.

\section{Generating subnets}

To derive all subnets with a given number of inequivalent nodes for a particular net we have used the two-step algorithm proposed by Blatov (2007). Starting from an initial threeperiodic net $N$ with a space group $G$ we considered all ways of decreasing its symmetry following the list of all translationequivalent and class-equivalent subgroups of $G$ that kept the number of inequivalent nodes of $N$. Then we enumerated all ways of breaking edges in $N$ as well as in its low-symmetry mappings. As a result, nets of different periodicity were obtained; we kept only three-periodic nets. If the resulting subnet had a Euclidean embedding with a higher space-group symmetry [as can be proved with the Systre program (Delgado-Friedrichs \& O'Keeffe, 2003)], the generating procedure was repeated for the most symmetric mapping of the subnet. In this case, some additional topologies were obtained as subnets of this symmetrized mapping; these could not be derived directly from the initial net. By removing some sets of edges from the initial net we obtain such subnets only in a low-symmetry embedding with a larger number of inequivalent nodes than in the initial net, so an additional step of symmetrization of the subnet embedding is always required. We shall call such subnets indirectly generated, unlike directly generated subnets produced by one step of the generating procedure. The number of indirectly generated subnets is rather large, for example among the 5278 uninodal nets with novel topologies described in the first paper of this series (Blatov, 2007) there are 969 such nets (18.3\%). However, the role of indirectly generated nets in crystal chemistry seems less important, since a more complex topological transformation leads to such nets (they have a small net relation graph degree, see $\$ 4$ below). The TOPOS program package and TTD collection were used to produce all subnets and to determine their topologies.

In the set of initial nets $N$ we included all 414 binodal nets with a node degree not larger than eight collected in the RCSR database (release of October 2007); among them there are 27 edge-transitive nets (Delgado-Friedrichs et al., 2006; Delgado-Friedrichs \& O'Keeffe, 2007). The eight edgetransitive nets with a higher node degree (3,12-coordinated ttt; 4,12-coordinated ftw, ith, shp; 6,12-coordinated alb, mgc; 3,24coordinated rht; 4,24-coordinated twf) were considered as well. Thus, we have examined all known binodal edgetransitive nets along with the most crystallochemically significant 3-8-coordinated binodal nets, as these coordinations are typical for metal-organic frameworks. Note that the RCSR database contains only 17 binodal nets that are not edgetransitive and have a node degree larger than eight (9-20). [These are $(9,20)$-alb-x, $(6,18)$-ast-d, $(12,14)$-bet, $(12,15)$-cla-d, (5,10)-fit, (3,9)-gfy, (10,12)-mbc, (12,16)-mgc-x, $(8,14)$-reo-d, (12,12)-tcj, (12,12)-tck, (12,12)-tcl, (12,12)-tcm, (10,10)-tcn, $(10,10)$-tco, $(9,20)$-tsl, $(3,9)$-xmz. Hereafter the node degrees $(n, m)$ are shown in parentheses before the symbol.]

From these 422 nets all subnets were derived (103 uninodal, which include 20 interpenetrated arrays, and 4024 binodal, of which 133 were interpenetrated), among which we found 3397 
Table 2

Binodal-quasi-uninodal nets.

Refined unit-cell dimensions and node positions were obtained using Systre. The length of contacts between nodes is $1 \AA$.

\begin{tabular}{|c|c|c|c|c|c|c|}
\hline $\begin{array}{l}\text { Initial } \\
\text { net }\end{array}$ & $\begin{array}{l}\text { Transformation sequence and } \\
\text { resulting space group }\end{array}$ & $\begin{array}{l}\text { Node degrees, } \\
D\left(n: N_{n}\right)\end{array}$ & $\begin{array}{l}\text { Net name, } \\
\text { Transitivity } p q \dagger\end{array}$ & $\begin{array}{l}\mathbf{a}(\AA), \\
x 1, \\
x 2\end{array}$ & $\begin{array}{l}\mathbf{b}(\AA) / \beta\left(^{\circ}\right), \\
y 1, \\
y 2\end{array}$ & $\begin{array}{l}\text { c }(\AA), \\
z 1, \\
z 2 \\
\end{array}$ \\
\hline cbo-e & $P a \overline{3}$ & $\begin{array}{l}3,3 \\
(6: 78)\end{array}$ & $\begin{array}{l}\text { cbo-e- } 3,3-P a \overline{3}^{\ddagger} \\
23\end{array}$ & $\begin{array}{l}4.000 \\
0.2502 \\
0.0418\end{array}$ & $\begin{array}{l}4.000 \\
0.2918 \\
0.4925\end{array}$ & $\begin{array}{l}4.000 \\
0.4949 \\
0.2480\end{array}$ \\
\hline mbc & $\mathrm{Cmcm} \rightarrow C 222_{1} \rightarrow P 2_{1} 2_{1} 2(\mathbf{b}, \mathbf{c}, \mathbf{a} ; 1 / 4,0,0)$ & $\begin{array}{l}7,7 \\
(1: 7)\end{array}$ & $\begin{array}{l}\text { mbc- }-7,7-P 2_{1} 22 \\
28\end{array}$ & $\begin{array}{l}5.297 \\
0.6786 \\
0.4286\end{array}$ & $\begin{array}{l}1.308 \\
0.2500 \\
0.2500\end{array}$ & $\begin{array}{l}1.000 \\
0.0027 \\
0.5027\end{array}$ \\
\hline mbc & $\mathrm{Cmcm} \rightarrow \mathrm{Cmc2}_{1} \rightarrow \mathrm{Pca2}_{1}(-\mathbf{b}, \mathbf{a}, \mathbf{c} ; 1 / 4,1 / 4,0)$ & $\begin{array}{l}9,9 \\
(1: 9)\end{array}$ & $\begin{array}{l}\text { mbc- } 9,9-P c a 2_{1} \\
29\end{array}$ & $\begin{array}{l}5.975 \\
0.9408 \\
0.1775\end{array}$ & $\begin{array}{l}1.000 \\
0.7500 \\
0.7500\end{array}$ & $\begin{array}{l}1.000 \\
0.2500 \\
0.2500\end{array}$ \\
\hline tcj & $\begin{array}{l}P 6_{3} / m m c \rightarrow \text { Cmcm }(-\mathbf{a}-\mathbf{b}, \mathbf{a}-\mathbf{b}, \mathbf{c}) \rightarrow \operatorname{Pnma}(\mathbf{b}, \mathbf{c}, \mathbf{a}) \rightarrow \\
\quad P n a 2_{1}(\mathbf{a},-\mathbf{c}, \mathbf{b})\end{array}$ & $\begin{array}{l}9,9 \\
(1: 9)\end{array}$ & $\begin{array}{l}\text { tcj-9,9-Pna2 }{ }_{1} \neq \\
29\end{array}$ & $\begin{array}{l}1.732 \\
0.6667 \\
0.5000\end{array}$ & $\begin{array}{l}1.000 \\
0.0000 \\
0.5000\end{array}$ & $\begin{array}{l}3.266 \\
0.2500 \\
0.5000\end{array}$ \\
\hline tco & Cccm $\rightarrow$ Ama2 $(\mathbf{c}, \mathbf{a}, \mathbf{b} ; 0,0,1 / 4) \rightarrow \operatorname{Pna}_{1}(0,1 / 4,0)$ & $\begin{array}{l}9,9 \\
(1: 9)\end{array}$ & $\begin{array}{l}\text { tco-9,9-Pna } 2_{1} \ddagger \\
29\end{array}$ & $\begin{array}{l}3.821 \\
0.2790 \\
0.0290\end{array}$ & $\begin{array}{l}1.000 \\
0.0963 \\
0.2500\end{array}$ & $\begin{array}{l}1.674 \\
0.2767 \\
0.1259\end{array}$ \\
\hline
\end{tabular}

$\dagger p$ and $q$ are the numbers of different kinds of topologically non-equivalent nodes and edges in the net (cf. Ockwig et al., 2005). $\ddagger$ Binodal sphere packing.

topologically distinct nets $(82.3 \%)$ not contained in the databases EPINET, RCSR and TTD, including 496 indirectly generated nets $(14.6 \%)$. All the new nets but one correspond to binodal nets; the only novel uninodal subnet is 3coordinated with vertex symbol $\left[6.12_{4} \cdot 12_{4}\right]$. No new edgetransitive nets were revealed. To designate subnets we have used $\mathbf{s}-d-G-n$ symbols (Blatov, 2007), where $\mathbf{s}$ is the conventional name of the initial net, $d$ is a set of ascending integers equal to the degrees of all inequivalent nodes in the subnet, $G$ is the space group for the most symmetrical embedding of the subnet and $n$ is optional and enumerates non-isomorphic subnets with a given $\mathbf{s}-d-G$ sequence. For example, the symbol stc-5,6-C2/m-1 encodes a 5,6-coordinated subnet of the 6,6coordinated RCSR net stc; the highest possible symmetry of the net in Euclidean space is $C 2 / m$; there are other stc subnets with the same symmetry and node degrees; this one is the first net in the list. ${ }^{2}$ Fig. 1 illustrates the procedure for deriving two subnets from $(4,6)$-fsc (see also Table 5 below); both subnets with the embedding of the original net as well as the Systreoptimized net are compared with one real example. Here we also show one case where the space group/embedding of the net in Systre (fsc-3,5-P4/mbm) differs from the one directly derived by the removal of some edges (fsc-3,5-Pnma): moreover the real structures often have another embedding (with

\footnotetext{
2 The crystallographic data for all 99 new binodal nets mentioned in this paper are available as a TOPOS database and a text file in Systre input format from the IUCr electronic archives (Reference: EO5002). Services for accessing these data are described at the back of the journal.
}

different space group, as for DOQRUW) but the underlying topology is preserved.

We consolidated all the initial nets with their subnets in a net relation graph (NRG), which shows pathways linking different topologies (Blatov, 2007). In total there are 4127 nets in the NRG, 3974 single and 153 interpenetrated, 4024 binodal and 103 uninodal, including the 3397 novel nets $(82.3 \%)$. Most of the known 577 single nets $(14.5 \%)$ are listed in the RCSR database (334), but there are also 225 EPINET nets (Table 1) and 18 TTD nets not reported among the RCSR nets [AFUQOH, $\mathrm{B}_{2} \mathrm{O}_{3}$ (high pressure) (34685), $\mathrm{CaCl}_{2}$ (86209), $\mathrm{Cs}_{2} \mathrm{Se}$ (41687), $\mathrm{Cu}_{2} \mathrm{~S}$ (16550), $\mathrm{Cu}_{3} \mathrm{As}$ (100149), ENCDNB01, INIQUR, LEJCAE, $\mathrm{Mg}_{2} \mathrm{C}_{3}$ (71941), NiP (27159), $\mathrm{ScD}_{0.33}$ (46032), $\mathrm{SiO}_{2}$ (56684), $\mathrm{SnF}_{2}$ (14194), $\mathrm{TiSi}_{2}$ (96029), two uninodal sphere packings $(3 / 8 / c 3$ and $3 / 8 / t 7)$ and the minimal net 4(3)4]. [If a TTD net occurs in a real structure, it is identified by a refcode or collection code from the Cambridge Structural Database (CSD) or the Inorganic Crystal Structure Database (ICSD), respectively. Sphere packings are considered in detail by Koch et al. (2006) and minimal nets by Beukemann \& Klee (1992) and Bonneau et al. (2004). All the TTD nets are simplified structures of inorganic or metal-organic frameworks; the simplification corresponds to the so-called standard representation (see $\$ 3$ ).]

We have also treated all ten non-edge-transitive binodal RCSR nets containing 9-12-coordinated nodes; among them there are 12-coordinated close sphere packings [tcj $(h c)$, tck $(h c c)$, tcl $(h h c)$ and tem $(h h c c)]$. As expected, the number of subnets for these nets is much larger; many of them have 
combinations of node degrees that are unusual for chemical compounds, for example $(7,8),(9,11)$ etc. For this reason the remaining seven binodal RCSR nets with node degrees larger than 12 (alb-x, ast-d, bet, cla-d, mgc-x, reo-d, tsl) were not considered. Moreover, only directly generated subnets were analyzed for the ten 9-12-coordinated nets and among them there are 34907 novel topologies. These topologies were not included in the NRG since most of them are not so important for crystal chemistry, otherwise they would have appeared among the subnets generated from the 422 nets in the sample used. Nonetheless, all the $3397+34907=38304$ novel topologies have been stored in the TTD collection and are available at http://www.topos.ssu.samara.ru. Among them there are $5959 n$-regular binodal nets $(15.5 \%)$ with the same coordination for both nodes $(273,586,639,1365,1246,1181$, 468, 178 and 23 nets for the $n, n$-coordination, $n=3-11$ respectively); we may also call them coordinatively uninodal or homocoordinated. In some exceptional cases $n$-regular binodal nets have a stronger similarity with uninodal nets, as can be seen if we consider other net invariants.

\section{Degree of similarity between nodes: the rare binodal-quasi-uninodal nets}

Following Blatov (2007), to determine the net topology we have used three invariants: (i) the coordination sequence $\left\{\mathrm{CS}_{k}\right\}, k=1-10$; (ii) the vertex symbol collecting the size and number of shortest rings (cycles that are not sums of two smaller cycles) meeting at angles of nodes; (iii) the vertex symbol for cycles collecting the size and number of shortest

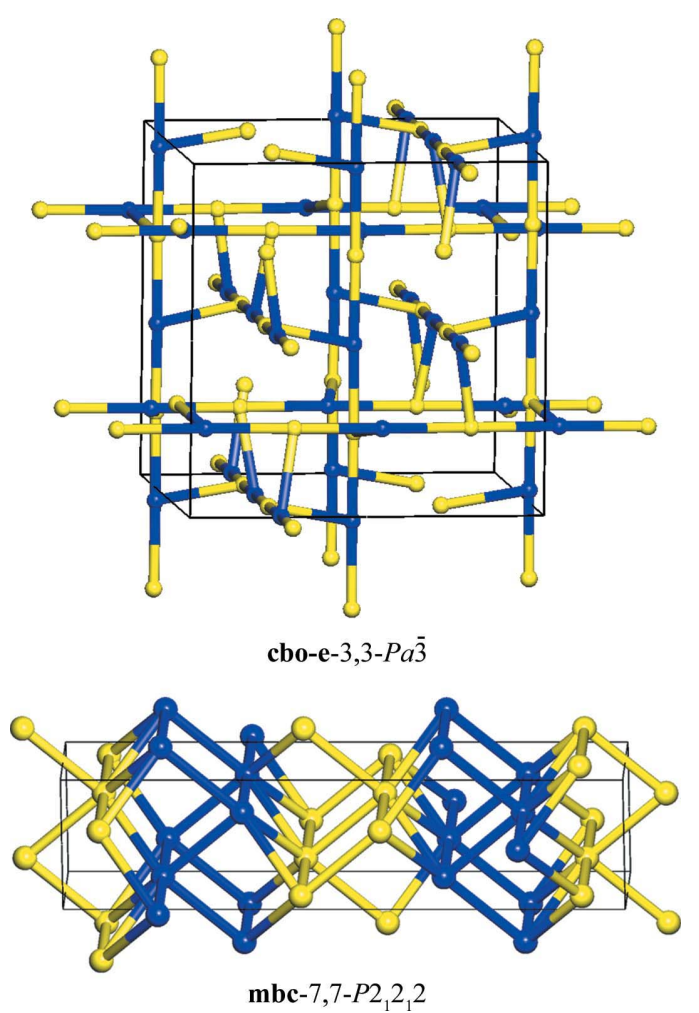

Figure 2

Two examples of the binodal-quasi-uninodal nets from Table 2. cycles in the net. As mentioned by Blatov (2007), no examples of non-isomorphic nets are known with all the invariants equivalent. However, we have discovered six $n$-regular binodal nets with two topologically different nodes that have the same set of the three invariants and so very similar local topologies. Thus, it is reasonable to call them binodal-quasiuninodal; they are gathered in Table 2. We also add the transitivity $p q$, where $p$ and $q$ are the numbers of different kinds of topologically non-equivalent nodes and edges in the net ( $c f$. Ockwig et al., 2005) as a rough measure of the complexity of the net; for example, edge-transitive nets have small transitivity values of 11 or 21 .

The topological difference between the nodes in the six nets was found with Systre, which uniquely proves the isomorphism of crystallographic nets without collisions (Delgado-Friedrichs \& O'Keeffe, 2003). The topological difference can also be demonstrated with one more invariant, the cycle sequence (Beukemann \& Klee, 1994; Thimm \& Klee, 1997), which extends the vertex symbol for cycles to all cycles up to a given size and can be computed with TOPOS. $^{\mathbf{3}}$ To study the nets in more detail, and to find a new invariant, we have implemented in TOPOS a procedure for searching for any kind of finite subgraph in a net; this procedure was used to compare shell graphs for inequivalent nodes. A shell graph $\mathrm{SG}_{n}$ is a graph obtained by successive growth of $n$ coordination shells around a given node; it consists of $N_{n}=\sum_{k=1}^{n} \mathrm{CS}_{k}$ nodes plus the origin and is unambiguously determined by its adjacency matrix. The adjacency matrix of a shell graph is a stronger invariant than the invariants mentioned above; the isomorphism of $\mathrm{SG}_{n}$ for given nodes means that the nodes are topologically equal within the first $n$ shells; for complete isomorphism the nodes are topologically equivalent up to $n=$ $\infty$. The degree of similarity $(D)$ for the nodes can be expressed as $n: N_{n}$, where both numbers relate to the largest equal shell graph; the larger both numbers are, the closer the similarity. In this respect, the most similar are the nodes in cbo-e-3,3-Pa $\overline{3}$ with $D=(6: 78)$ (Table 2, Fig. 2), i.e. their shell graphs are locally isomorphous within the first six coordination shells and contain $N_{6}+1=79$ nodes; the strict similarity in the other nets is limited by the first coordination shell, $n=1, D$ $=(1: N)$. Obviously, the quantity $D$ can also be used to compare nets; the larger the $D$ values for corresponding nodes of the nets are, the more similar are the nets. Let us emphasize that unlike the invariants relating to cycles, the degree of similarity can be used not only to find the net isomorphism, but also to explore the local topological sameness of nets.

For the moment, binodal-quasi-uninodal nets are unknown in crystals; however, binodal nets with a high $D$ exist. Probably the most exciting example is the crystal structure of bis $\left(\mu_{3^{-}}\right.$ imidazole-4,5-dicarboxylato)-( $\mu_{2}-4,4^{\prime}$-bipyridine $)$-diaqua-trizinc (XECBOX, Fig. 3a) (Lu et al., 2006). Considering $\mathrm{Zn}$ atoms and centroids of ligands as nodes we obtain a net (Fig. $3 b$ ) that can be simplified further by removing dangling water molecules and converting bridge bipyridine and $\mathrm{Zn}$ nodes into

\footnotetext{
${ }^{3}$ The cycle sequences computed with TOPOS for the six nets are given in the supplementary material.
} 
edges (Fig. 3c). We call this simplified representation of a framework standard because it naturally follows the chemical description of coordination compounds made of metals plus ligands, so nodes are assigned both to metals and ligands.

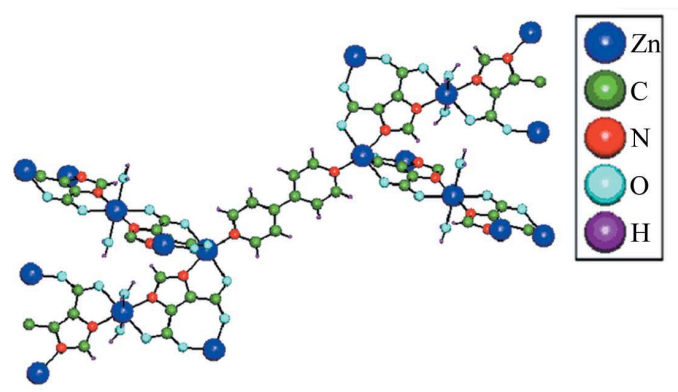

(a)

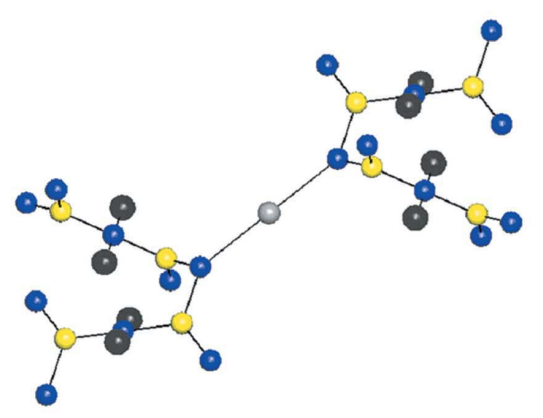

(b)

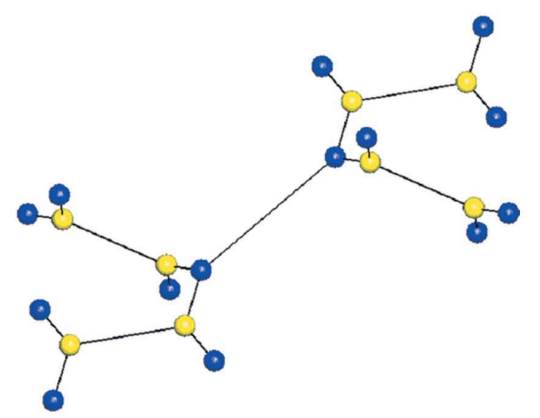

(c)

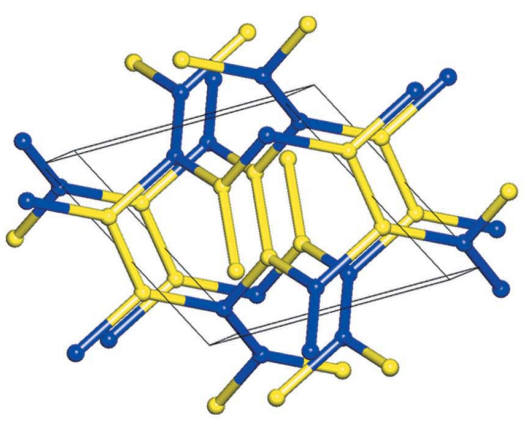

(d)

Figure 3

Different representations of the XECBOX crystal structure. (a) Initial framework; $(b)$ simplified net with the centroids of imidazole-4,5dicarboxylato (yellow balls), 4,4'-bipyridine (grey ball) and aqua (black balls) ligands; (c) standard representation as mbc-3,3- $P 2_{1} / c$ obtained from (b) by removing dangling water ligands and contracting bridge bipyridine and $\mathrm{Zn}$ nodes into edges; $(d)$ idealized mbc-3,3-P2 $/ c$ net.
Table 3

The binodal nets with large NRG degrees $(>50)$.

\begin{tabular}{|c|c|c|c|}
\hline Net & Transitivity $p q$ & Node degrees & NRG degree \\
\hline cbs & 26 & 5,7 & 386 \\
\hline sqc374 & 26 & 4,6 & 250 \\
\hline alb $\dagger$ & 21 & 6,12 & 228 \\
\hline $\mathbf{f s g}(s q c 10) \dagger$ & 24 & 4,6 & 187 \\
\hline cbs-5,6-Cmmm & 26 & 5,6 & 184 \\
\hline cbs-4,7-Cmmm & 26 & 4,7 & 175 \\
\hline stc $\ddagger$ & 23 & 6,6 & 169 \\
\hline lib $\ddagger$ & 24 & 6,6 & 144 \\
\hline crs-d $(s q c 870)$ & 22 & 4,8 & 134 \\
\hline hbr $\ddagger$ & 23 & 8,8 & 124 \\
\hline cor-e $\ddagger$ & 28 & 7,7 & 102 \\
\hline $\operatorname{seh} \ddagger$ & 22 & 6,8 & 102 \\
\hline cbs-5,6-Pmma & 27 & 5,6 & 99 \\
\hline fit-e $\neq$ & 23 & 7,8 & 98 \\
\hline cbs-5,6-Pmna & 27 & 5,6 & 96 \\
\hline nbo-x-d & 22 & 8,8 & 95 \\
\hline cbs-4,7-Pmna & 27 & 4,7 & 92 \\
\hline cbs-4,7-Pmma & 27 & 4,7 & 89 \\
\hline fsx & 23 & 5,6 & 88 \\
\hline sqc1067ま & 24 & 5,6 & 83 \\
\hline fsc $(s q c 11) \dagger$ & 22 & 4,6 & 78 \\
\hline crb-e & 23 & 6,6 & 73 \\
\hline stc-5,6- $C 2 / m-1 \ddagger$ & 25 & 5,6 & 73 \\
\hline stc- $5,6-C 2 / m-2 \ddagger$ & 25 & 5,6 & 73 \\
\hline cbs- $4,5-C m m m \ddagger$ & 25 & 4,5 & 71 \\
\hline scu $(s q c 170) \dagger \hbar$ & 21 & 4,8 & 68 \\
\hline rho-e $\ddagger$ & 24 & 6,6 & 65 \\
\hline neb-e $\$$ & 24 & 6,6 & 62 \\
\hline gis-e $\ddagger$ & 24 & 6,6 & 59 \\
\hline$s q c 280$ & 25 & 4,5 & 58 \\
\hline
\end{tabular}

$\dagger$ Occurs in crystal structures. $\$$ Binodal sphere packing.

Standard representations can be obtained by TOPOS in an automated mode. The resulting net is 3,3-coordinated binodal with nodes corresponding to imidazole-4,5-dicarboxylato ligands and $\mathrm{Zn}$ atoms. It is among the NRG nets and can be derived from the $(10,12)$-mbc net, hence the symbol is mbc$3,3-P 2_{1} / c$. Both vertex symbols (for rings and for cycles) for the two nodes are $\left[10.10 .10_{3}\right]$, but coordination sequences differ starting from the eleventh shell, so the net is not binodal-quasi-uninodal. However, $D=9: 702$, so the nodes are locally strongly similar, up to the ninth shell, and play almost the same role in the net.

\section{Topological relations between binodal nets}

As mentioned by Blatov (2007), the crystallochemical significance of a net is reflected in the role that the net plays in the NRG. If other factors are equal, the nets with large NRG degrees have a higher probability of occurring in nature because they are related to many other nets and have a lot of ways to be transformed to/from other topologies. In Table 3 the single nets with the largest NRG degrees $(>50)$ are collected and in Fig. 4 we show the embeddings for the first six. Note that, compared to uninodal nets (Blatov, 2007), interpenetrated arrays are not as significant in the NRGs of binodal nets; the largest degree (40) belongs to (3,6)-sit twofold interpenetrated.

As for uninodal nets, the occurrence of binodal nets depends on the node degree; the most frequent combinations 


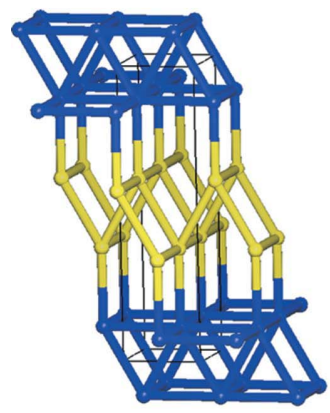

$(5,7)-\operatorname{cbs}$

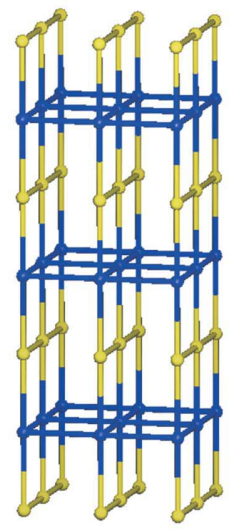

$(4,6)-$ fsg

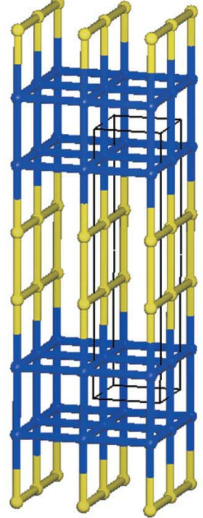

$(4,6)-s q c 374$

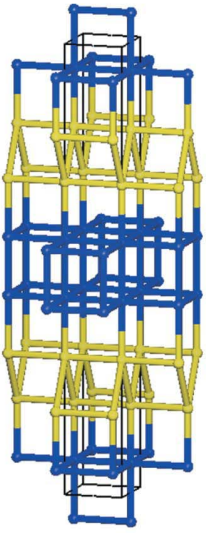

cbs-5,6-Cmmm

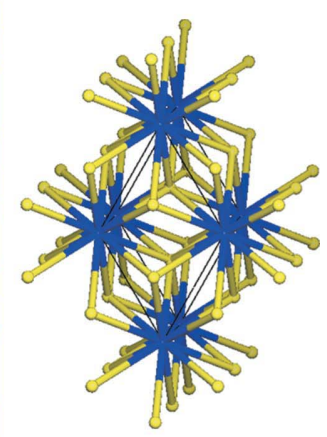

$(6,12)$-alb

Figure 4

Six Systre-idealized nets with highest NRG degree taken from Table 3.

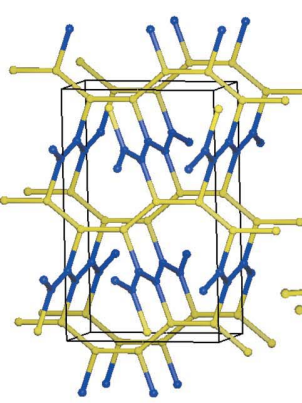

cbs-3,3-Cmcm

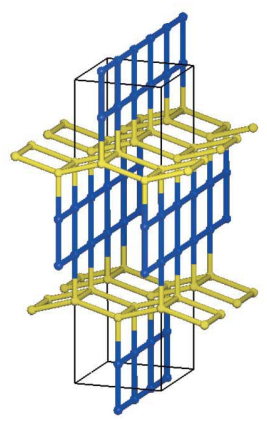

cbs-4,4-Imma-1

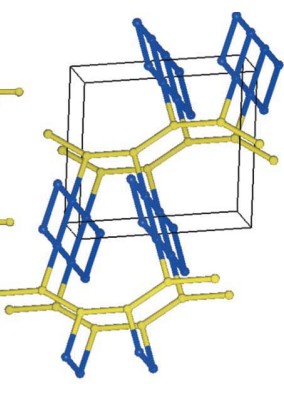

cbs-3,4-Pmma

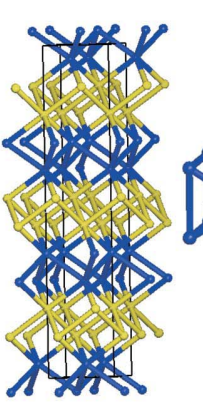

$(6,6)-c b s$

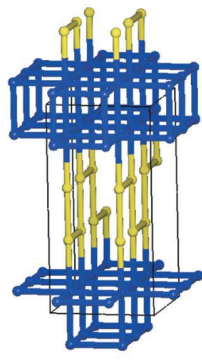

cbs-3,6-Cmme

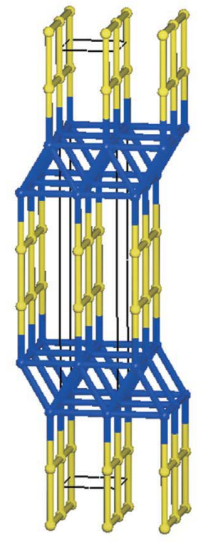

cbs-4,7-Cmmm

typical for metal-organic frameworks correspond to small node degrees of 3, 4 or 6. Many nets in Table 3 have other node degrees, therefore in Table 4 we collect the first ten nets with the largest NRG degrees for some typical coordinations and in Fig. 5 we show one net for each coordination. Note that among the nets in Tables 3 and 4 there are many binodal sphere packings, i.e. embedding of the type $1 \mathrm{a}$ or $1 \mathrm{~b}$ (DelgadoFriedrichs \& O'Keeffe, 2005). Let us emphasize that unlike uninodal sphere packings, which have been intensively studied for years [Koch et al. (2006) and references therein], polynodal sphere packings have not been considered in the literature, although some of them are collected in the RCSR database. There are obvious correspondences between nets in Tables 3 and 4: many nets with novel topologies are subnets of the nets with a large NRG degree, such as cbs, fsc, fsg, seh, stc. Thus, some nets can be crystallochemically significant indirectly: the nets themselves do not occur in nature, but their subnets do. At the same time, some nets with a large NRG degree do not
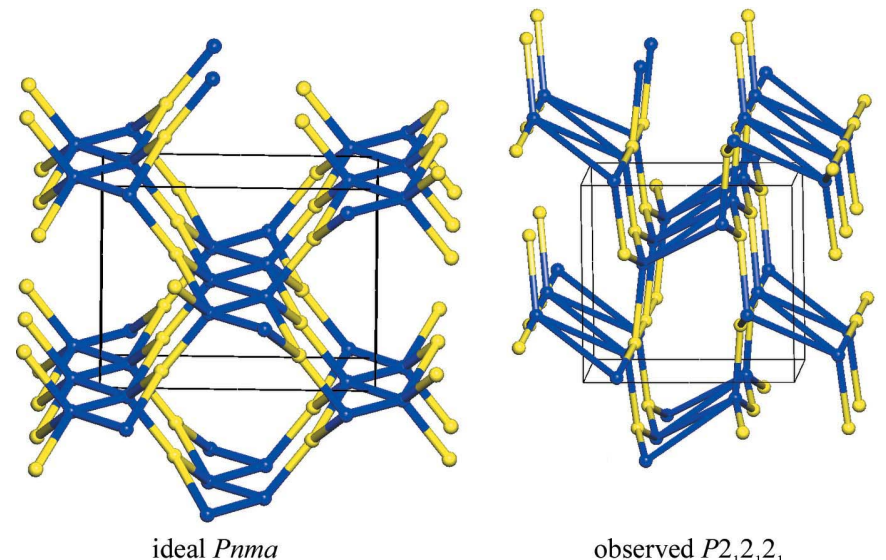

crs-d-3,5-Pnma

observed $P 2{ }_{1} 2_{1} 2_{1}$

(a)

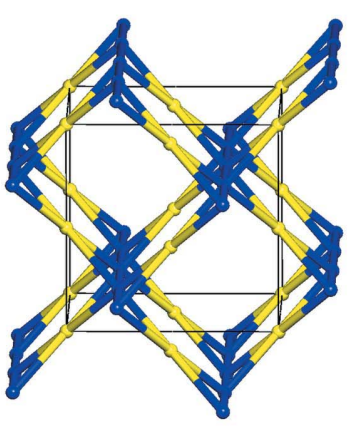

ideal Imma

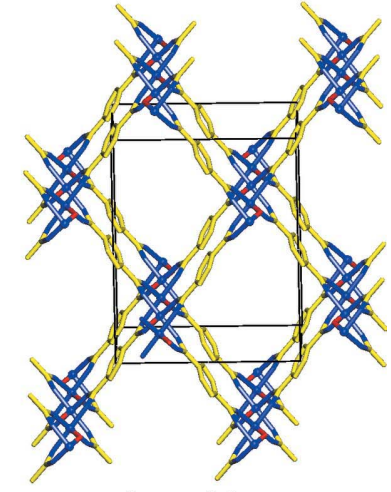

observed Imma

seh-4,6-Imma

(b)

Figure 6

(a) The ideal crs-d-3,5-Pnma net with the one observed in four (cobalamine) $X(\mathrm{LiCl})_{2}, X=\mathrm{N}^{3-}, \mathrm{Cl}^{-}, \mathrm{NO}^{3-}, \mathrm{CN}^{-}$(refcodes GIZYOD, GIZYUJ, HUSPAM, WIKXUJ); the 5-coordinated node represents the cobalamine and the 3-coordinated node represents the Li. (b) The ideal seh-4,6-Imma net with the one observed in two $M(\mathrm{OH})(1,4$-benzenedicarboxylato) complexes, $M=\mathrm{Cr}^{3+}, \mathrm{Al}^{3+}$ (refcodes MINVUA, SABVUN; the bridging oxygen is shown in red).

Six selected representatives of 3, 4 and 6-coordinated nets from Table 4. 
Table 4

A selection of binodal nets with node degrees 3, 4 or 6 .

Node degrees 3, 4 or 6 are typical for coordination compounds. The ten nets with the largest NRG degree for each class are listed.

\begin{tabular}{|c|c|c|c|c|c|c|c|c|}
\hline Net & $\begin{array}{l}\text { Transitivity } \\
p q\end{array}$ & $\begin{array}{l}\text { NRG } \\
\text { degree }\end{array}$ & Net & $\begin{array}{l}\text { Transitivity } \\
p q\end{array}$ & $\begin{array}{l}\text { NRG } \\
\text { degree }\end{array}$ & Net & $\begin{array}{l}\text { Transitivity } \\
p q\end{array}$ & $\begin{array}{l}\text { NRG } \\
\text { degree }\end{array}$ \\
\hline \multicolumn{3}{|c|}{ 3,3-coordinated (3-regular) nets } & \multicolumn{3}{|l|}{ 3,6-coordinated nets } & \multicolumn{3}{|c|}{ 4,6-coordinated nets } \\
\hline cbs-3,3-Cmcm ${ }^{\dagger}$ & 24 & 28 & cbs-3,6-Cmme $\dagger$ & 27 & 37 & $s q c 374$ & 26 & 250 \\
\hline baf-3,3-I4/mmm & 23 & 27 & ant $† \neq$ & 22 & 27 & fsg $(s q c 10) \ddagger$ & 24 & 187 \\
\hline baf-3,3-Imma & 24 & 26 & cbs-3,6-Cmmm-1 & 25 & 20 & fsc $(s q c 11) \neq$ & 22 & 78 \\
\hline cbs $-3,3-C 2 / m-2 \dagger$ & 25 & 23 & cbs-3,6-Cmmm-2 & 26 & 19 & stc- $4,6-P \overline{3} m 1 \dagger$ & 23 & 46 \\
\hline bbe-3,3-Imma $\dagger$ & 23 & 22 & mgc- $3,6-I 4_{1} 22$ & 22 & 18 & stc- $4,6-R \overline{3} m$ & 23 & 46 \\
\hline cbs-3,3-Pmna $\dagger$ & 25 & 21 & rtl $+\neq$ & 22 & 16 & cbs-4,6-Pmma & 26 & 36 \\
\hline $\operatorname{iph} \dagger$ & 23 & 21 & $s q c 251$ & 25 & 16 & cbs-4,6-Pmmn & 26 & 33 \\
\hline fsg-3,3-Fddd† & 23 & 17 & $s q c 2983$ & 25 & 16 & cbs-4,6-Imma & 27 & 33 \\
\hline nof $\dagger$ & 23 & 17 & cbs-3,6-Cmcm-1 & 26 & 15 & fsd $(s q c 61) \dagger$ & 23 & 32 \\
\hline fry $-3,3-C 222 \dagger$ & 25 & 16 & cbs-3,6-Cmcm-2 & 26 & 15 & $s q c 502 \dagger \div$ & 22 & 32 \\
\hline \multicolumn{3}{|c|}{ 3,4-coordinated nets } & \multicolumn{3}{|c|}{ 4,4-coordinated (4-regular) nets } & \multicolumn{3}{|c|}{ 6,6-coordinated (6-regular) nets } \\
\hline cbs-3,4-Pmma & 25 & 38 & cbs-4,4-Imma-1† & 26 & 19 & stc $\dagger$ & 23 & 169 \\
\hline cbs-3,4-Cmmm-1 & 25 & 28 & cbs-4,4-Cmcm $\dagger$ & 26 & 16 & $\operatorname{lib} \dagger$ & 24 & 144 \\
\hline cbs-3,4-Cmmm-2 & 24 & 25 & baf $\dagger$ & 25 & 15 & crb-e $\dagger$ & 23 & 73 \\
\hline fry & 25 & 24 & ful $\dagger$ & 27 & 14 & rho-e $\dagger$ & 24 & 65 \\
\hline tfi $(s q c 515) \dagger \ddagger$ & 22 & 23 & mog-e-x-z-4,4- $I 4 / \mathrm{mmm}^{\dagger}$ & 24 & 14 & neb-e $\dagger$ & 24 & 62 \\
\hline bbe-3,4-Cmmm & 23 & 22 & bcp $\dagger$ & 24 & 13 & gis-e $\dagger$ & 24 & 59 \\
\hline cbs-3,4-Cmme $\dagger$ & 25 & 20 & bcq $(s q c 941) \dagger$ & 24 & 13 & sta $\dagger$ & 22 & 48 \\
\hline cbs-3,4-Fmmm $\dagger$ & 25 & 18 & fvq $\dagger$ & 27 & 13 & $\mathbf{s t b} \dagger$ & 22 & 48 \\
\hline$s q c 1255$ & 24 & 18 & $\operatorname{mot}(s q c 29) \ddagger$ & 22 & 13 & nia $†$ t & 21 & 39 \\
\hline$s q c 185 \dagger$ & 22 & 18 & pts $(s q c 183) \dagger+$ & 21 & 13 & $\operatorname{mhg} \dagger$ & 25 & 29 \\
\hline
\end{tabular}

$\dagger$ Binodal sphere packing. $\$$ Occurs in crystal structures.

Table 5

Seventy-six metal-organic frameworks with the $(4,6)$-coordinated fsc and derived new topologies.

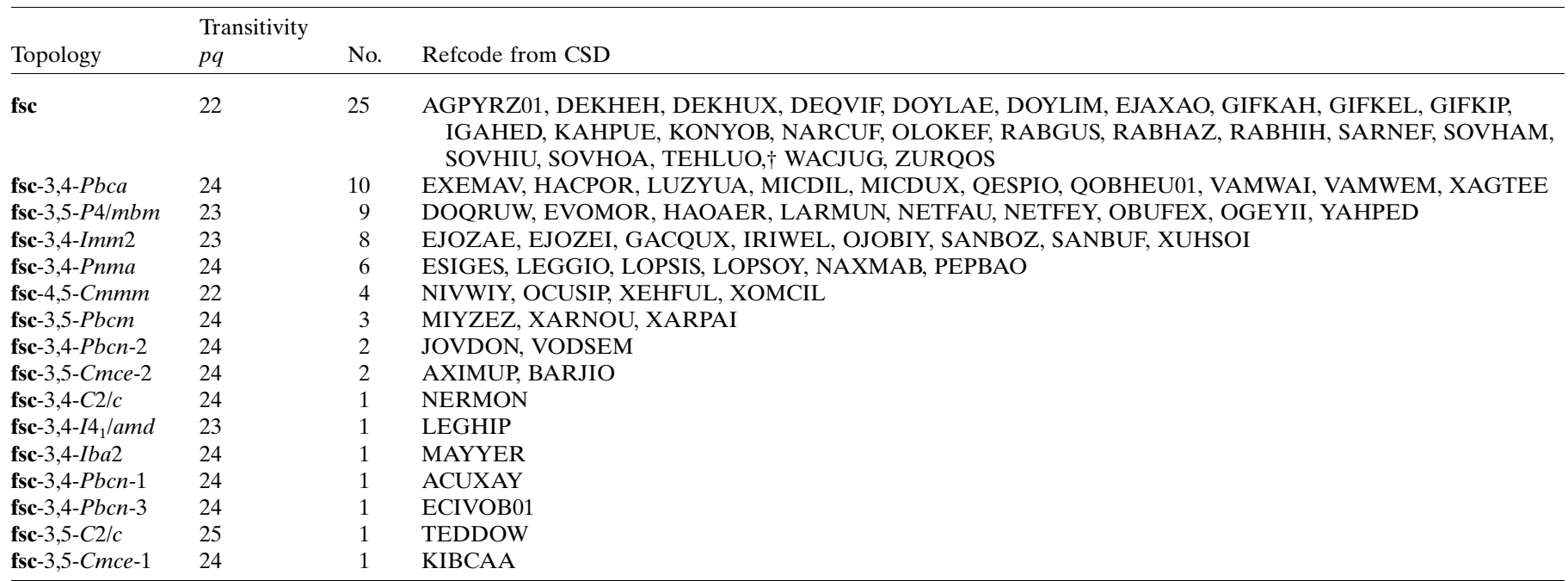

$\dagger$ Obtained by 'cluster representation'.

produce crystallochemically significant subnets of novel topology; among them there are sqc374, lib and hbr.

\section{Occurrence of the binodal nets in metal-organic frameworks}

For the moment, there are no comprehensive data on the occurrence of binodal nets in crystal structures. Ockwig et al.
(2005) listed examples of $n$-regular binodal nets and found more than one example for the 3-coordinated (3-regular) nets nof and noj, 4-coordinated pts and mog, 5-coordinated nok and 6-coordinated nia; four of them, nof, pts, nok and nia, are among the significant NRG nets (see Table 4; indeed nok occupies the fourth place among the 5,5-coordinated nets). To assess the occurrence of other binodal nets, the total file of crystallographic data on inorganic and metal-organic frame- 
Table 6

Eleven hydrogen-bonded and 102 metal-organic frameworks with topologies of some new subnets derived from nets with a large NRG.

The refcodes for hydrogen-bonded frameworks are given in italics.

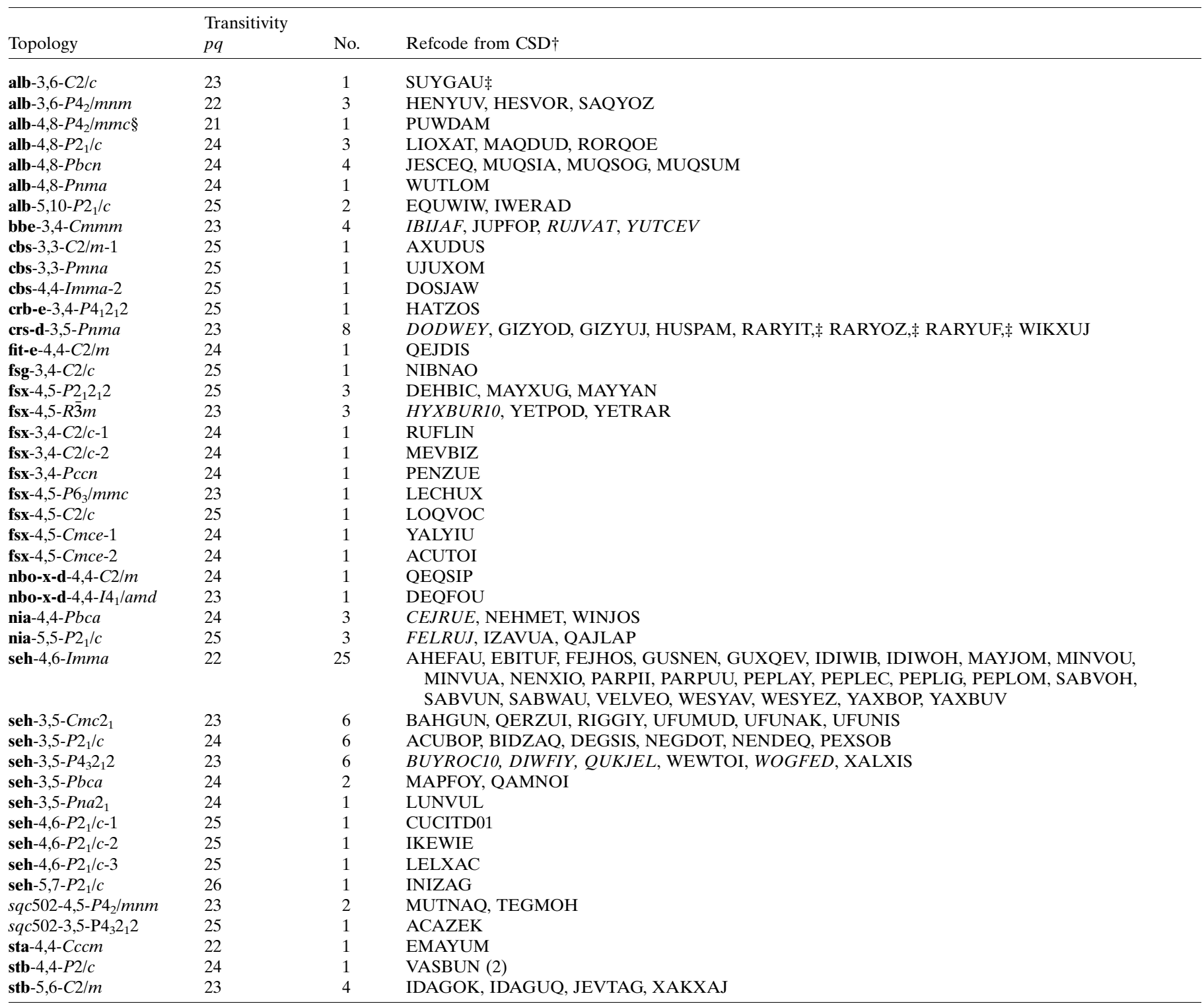

$\dagger$ For interpenetrated arrays the number of nets is given in parentheses. $\$$ Obtained by 'cluster representation'. $\S$ Net with collisions.

works should be processed. This would be a large task; here we consider only some examples of the NRG binodal nets found in organic and metal-organic frameworks. To find the examples reported in Tables 5-7 we have automatically generated the standard representations for 2131 three-dimensional metal-organic polymeric frameworks and 2473 hydrogenbonded organic and metal-organic frameworks: their net topology identifications are currently contained in the TTO collection. If the standard representation gives unknown topology, we applied a newly designed TOPOS procedure for an automated search for clusters of nodes in augmented or decorated nets. After contracting such clusters to their centroids, the resulting net contains only the centroids of the clusters and edges connecting them; we will call this cluster representation (details on the procedure will be discussed in a future publication).

According to Table 3, many binodal nets with mixed coordination play a significant role in the NRG. Among them there are 4,6-coordinated nets not considered by Ockwig et al. (2005). Examples for the sqc374 net are still unknown, but we have found two fsg nets of hydrogen-bonded organic molecules (JIKHOA and THYMDN01), as well as two organic hydrogen-bonded (WELMEF and a threefold array in TIJKOM) and 25 metal-organic frameworks with the fsc topology (Table 5 and Fig. 1). It is important that many novel binodal nets derived from fsc have been revealed among crystal structures as well. This fact proves that the fsc motif plays a significant role in metal-organic frameworks. 
Table 7

Four hydrogen-bonded and 152 metal-organic frameworks with topologies of binodal edge-transitive nets.

The refcodes for hydrogen-bonded frameworks are given in italics.

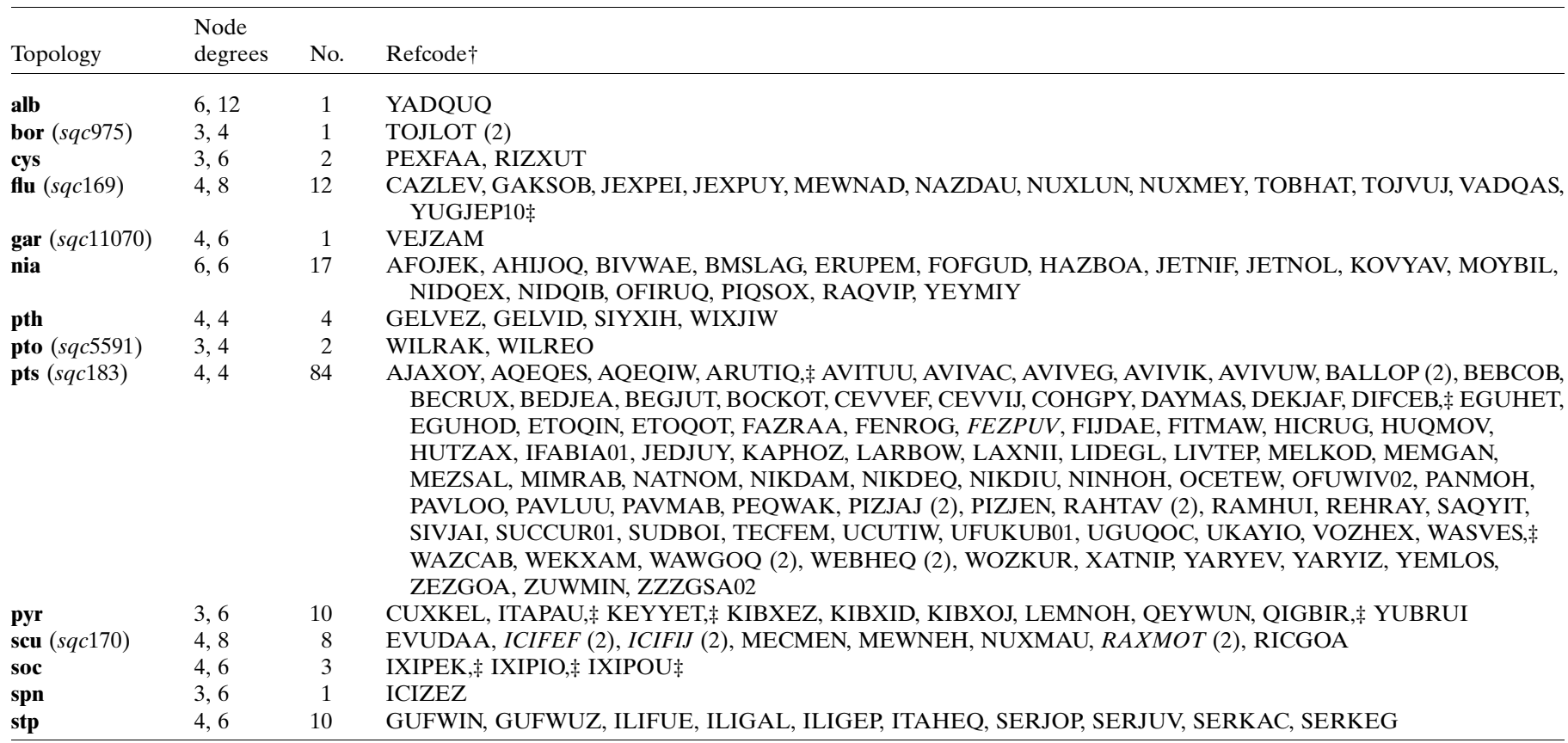

$\dagger$ For interpenetrated arrays the number of nets is given in parentheses. $末$ Obtained by 'cluster representation'.

Some nets with large NRG degrees have still not been found in crystal structures, but their subnets can be rather frequent (Table 6 and Fig. 6). It is relevant to note that all frequently occurring nets from Tables 3-6 have small (21-24) transitivities, which is in agreement with the ideas of Ockwig et al. (2005). The nets producing many subnets from Tables 5 and 6 , such as fsc, fsx and seh, also obey this condition. Note an interesting example of a novel edge-transitive net but with collisions (alb-4,8-P4 $/ m m c$ ), which is closely related to $(4,4)$ pts, another edge-transitive net (see Fig. 7) [for more on nets with collisions see Carlucci et al. (2007) and DelgadoFriedrichs \& O'Keeffe (2003)].

Table 7 contains all known examples of binodal edgetransitive nets [13 out of 27 3-8-coordinated nets as well as $(6,12)$-alb] in metal-organic frameworks. Thus, the data confirm the assumption of Delgado-Friedrichs et al. (2006, 2007) about the importance of edge-transitive nets for crystal chemistry.

Many nets from Tables 3 and 4 have not been found in crystal structures so far, but not all known crystal structures
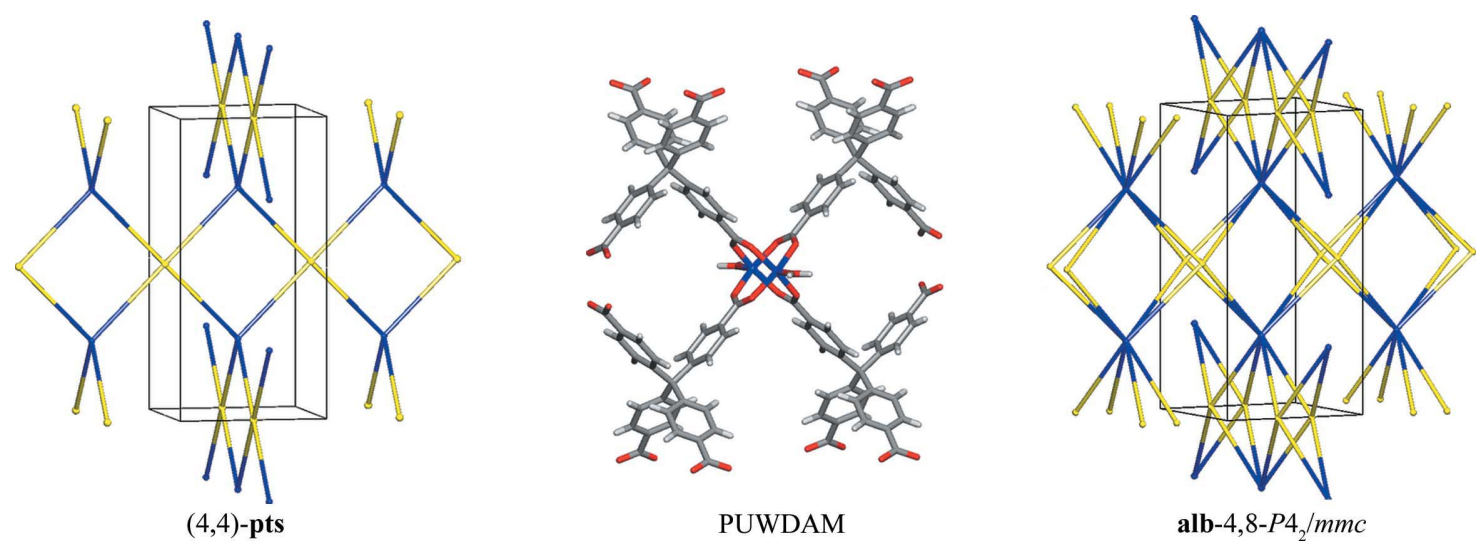

Figure 7

The two possible descriptions of the metal-organic framework observed in the crystal structure of PUWDAM, $\mathrm{Zn}_{2}$ [tetrakis(4-carboxyphenyl)methane]: on the left is the usually chosen edge-transitive $(4,4)$-pts net [nodes: $\mathrm{Zn}_{2}(\mathrm{COO})_{4}$ paddle wheel and the tetrahedral ligand]; on the right a different simplification that gives alb-4,8-P4 $/ \mathrm{mmc}$, a rare edge-transitive net with collisions (nodes: the octa-oxygen donor ligand as 8 -coordinated and the $\mathrm{Zn}$ as 4-coordinated). 
have been topologically investigated. Moreover, there are obviously other useful parameters related to the net global topology besides the node coordination, coordination figure, net transitivity and NRG degree mentioned by O'Keeffe et al. (2000), Ockwig et al. (2005) and Blatov (2007). Attention should be drawn to local topological features and to symmetry relations in the net (Baburin \& Blatov, 2007).

\section{Concluding remarks}

Our results show that the method for deriving subnets using space-group-subgroup relations is most general and, in principle, can be successfully applied to generate nets of any coordination. Many of the resulting subnets occur in crystal networks; the topological motifs that are important for crystal chemistry can be found by analysis of the experimental crystal data stored in electronic topological databases, taking into account the information on net-subnet relations. In fact, this approach opens a new phase in the development of crystal science: the search for overall structure correlations on the basis of all experimental data collected by structural chemistry. An important task at this stage is to extract the occurrence of nets in all crystal structures; a detailed analysis of the occurrence will be presented in a future article.

This work was supported by a Cariplo Fellowship to V. A. Blatov issued by Cariplo Foundation \& Landau Network Centro Volta (Como, Italy).

\section{References}

Baburin, I. A. (2008). Z. Kristallogr. 223, 371-381.

Baburin, I. A. \& Blatov, V. A. (2007). Acta Cryst. B63, 791-802.

Baburin, I. A., Blatov, V. A., Carlucci, L., Ciani, G. \& Proserpio, D. M. (2005). J. Solid State Chem. 178, 2452-2474.

Baburin, I. A., Blatov, V. A., Carlucci, L., Ciani, G. \& Proserpio, D. M. (2008a). Cryst. Growth Des. 8, 519-539.

Baburin, I. A., Blatov, V. A., Carlucci, L., Ciani, G. \& Proserpio, D. M. (2008b). Cryst. Eng. Commun. 10, 1822-1838.

Beukemann, A. \& Klee, W. E. (1992). Z. Kristallogr. 201, 37-51.

Beukemann, A. \& Klee, W. E. (1994). Z. Kristallogr. 209, 709-713.

Blatov, V. A. (2007). Acta Cryst. A63, 329-343.
Blatov, V. A., Carlucci, L., Ciani, G. \& Proserpio, D. M. (2004). Cryst. Eng. Commun. 6, 377-395.

Blatov, V. A. \& Peskov, M. V. (2006). Acta Cryst. B62, 457-466.

Bonneau, C., Delgado-Friedrichs, O., O'Keeffe, M. \& Yaghi, O. M. (2004). Acta Cryst. A60, 517-520.

Carlucci, L. Ciani, G. \& Proserpio, D. M. (2007). Making Crystals by Design. Methods, Techniques and Applications. Edited by D. Braga \& F. Grepioni, pp. 58-85. Darmstadt: Wiley.

Delgado-Friedrichs, O., Foster, M. D., O’Keeffe, M., Proserpio, D. M., Treacy, M. M. J. \& Yaghi, O. M. (2005). J. Solid State Chem. 178, 2533-2554.

Delgado-Friedrichs, O. \& O'Keeffe, M. (2003). Acta Cryst. A59, 351360.

Delgado-Friedrichs, O. \& O'Keeffe, M. (2005). J. Solid State Chem. 178, 2480-2485.

Delgado-Friedrichs, O. \& O'Keeffe, M. (2007). Acta Cryst. A63, 344 347.

Delgado-Friedrichs, O., O'Keeffe, M. \& Yaghi, O. M. (2006). Acta Cryst. A62, 350-355.

Delgado-Friedrichs, O., O'Keeffe, M. \& Yaghi, O. M. (2007). Phys. Chem. Chem. Phys. 9, 1035-1043.

Eon, J.-G. (2005). Acta Cryst. A61, 501-511.

Grünbaum B. \& Shephard G. C. (1987). Tilings and Patterns. New York: Freeman.

Hyde, S. T., Delgado-Friedrichs, O., Ramsden, S. J. \& Robins, V. (2006). Solid State Sci. 8, 740-752.

Klee, W. E. (2004). Cryst. Res. Technol. 39, 959-968.

Koch, E. \& Fischer, W. (1978). Z. Kristallogr. 148, 107-152.

Koch, E., Fischer, W. \& Sowa, H. (2006). Acta Cryst. A62, 152-167.

Lu, W. G., Jiang, L., Feng, X. L. \& Lu, T. B. (2006). Cryst. Growth Des. 6, 564-571.

Ockwig, N. W., Delgado-Friedrichs, O., O'Keeffe, M. \& Yaghi, O. M. (2005). Acc. Chem. Res. 38, 176-182.

Öhrström, L. \& Larsson, K. (2005). Molecule-Based Materials: The Structural Network Approach. Amsterdam: Elsevier.

O'Keeffe, M. (1992). Aust. J. Chem. 45, 1489-1498.

O'Keeffe, M., Eddaoudi, M., Li, H., Reineke, T. M. \& Yaghi, O. M. (2000). J. Solid State Chem. 152, 3-20.

O'Keeffe, M. \& Hyde, B. G. (1980). Philos. Trans. R. Soc. London, 295, 553-618.

O'Keeffe, M., Peskov, M. A., Ramsden, S. J. \& Yaghi, O. M. (2008). Acc. Chem. Res. 41, 1782-1789.

Ramsden, S. J., Robins, V. \& Hyde, S. T. (2009). Acta Cryst. A65, 81108.

Thimm, G. \& Klee, W. E. (1997). Zeolites, 19, 422-424.

Treacy, M. M. J., Rivin, I., Balkovsky, E., Randall, K. H. \& Foster, M. D. (2004). Microporous Mesoporous Mater. 74, 121-132.

Wells, A. F. (1977). Three-Dimensional Nets and Polyhedra. New York: Interscience. 